\title{
Evaluating the necessity of additional aquatic plant testing by comparing the sensitivities of different species
}

\author{
Marco Vervliet-Scheebaum ${ }^{1,2, *}$, Katja Knauer ${ }^{2}$, Steve J. Maund ${ }^{2}$, Reinhardt Grade ${ }^{2}$ \\ \& Edgar Wagner ${ }^{1}$ \\ ${ }^{1}$ Department of Plant Physiology, Institute of Biology II, Albert-Ludwigs-University, Schaenzlestrasse 1, D-79104 \\ Freiburg im Breisgau, Germany \\ ${ }^{2}$ Syngenta Crop Protection AG, CH-4002 Basel, Switzerland \\ (*Author for correspondence: E-mail: marco.vervliet@biologie.uni-freiburg.de)rcsobti@pu.ac.in
}

Key words: sensitivity, aquatic plants, herbicides, risk assessment

\begin{abstract}
At present, at least three and up to five plant species are required to assess the potential risks of herbicides to non-target aquatic plants. Several regulatory authorities are considering whether there should be further requirements based on concerns about the possible selectivity of herbicides (e.g., specific modes of action against dicotyledonous plants). The relative sensitivity of a range of aquatic plants is assessed in our work in order to evaluate the implications of differences in species sensitivity for aquatic risk assessment of herbicides. We therefore present results from ecotoxicological tests performed at Syngenta Crop Protection $\mathrm{AG}$ on various aquatic plants and compare them to available studies and results in literature. The criterion used for sensitivity ranking is the EC50 (median effect concentration) value, which allows a better comparison of values from different testing methods and conditions. The overall results obtained in the present work show that the aquatic risk assessment procedure for herbicides based on Lemna sp. and algae is sufficiently protective while identifying potential toxicity to non-target plants. Only few exceptions concerning herbicides with selective modes of action (e.g., auxin simulators) may require additional species testing for proper risk assessment.
\end{abstract}

\section{Introduction}

The preliminary risk assessment procedure for assessing the effects of herbicides on non-target aquatic plants requires data from a whole series of test organisms. For the EU, two species of freshwater algae (a green algae such as Selenastrum capricornutum Printz and a species from another taxonomic group e.g., the blue-green alga Anabaena flos-aquae (Lyngb) Bréb.) are tested. In addition, for US registration, data are required for a freshwater diatom (e.g., Naviculla pelliculosa (Bréb.) Hilse) and a marine alga (e.g., the diatom Skeletonema costatum Grev.). For both the EU and the US, data on the aquatic macrophyte Lemna sp. L. are also required.
A number of regulatory authorities are currently considering whether such a database is sufficient to adequately assess potential risks to aquatic plants. For example, the US EPA (2001) published a 'Proposal to Update Non-Target Plant Toxicity Testing under NAFTA' and concluded that aquatic macrophytes are under-represented. They propose that the number of vascular plants should be increased.

While evaluating the necessity for further aquatic plant testing, one should consider applying an appropriate uncertainty factor which could lead to a risk assessment that is sufficient to protect from harmful effects under field conditions.

In order to assess the potential impact of differences in species sensitivity on aquatic risk 
assessment for non-target aquatic plants, available data from our laboratory and from the literature were reviewed.

\section{Materials and methods}

Algal and macrophyte species used in the sensitivity ranking at Table 2 are presented by full name in Table 1.

A description of the various methods investigating the toxicity of herbicides on non-target aquatic plants can be found in Table 2 . The complete methodology of the experiments taken from literature can be found in Fairchild et al. (1998), Green \& Westerdahl (1990) and Netherland \& Getsinger (1992). The toxicity values referring to algae and Lemna sp. for the auxin simulating compounds are taken from Brock et al. (2000) and are based on the geometric mean of the available results in literature for each species. Toxicity values from required test species of the preliminary risk assessment (algae and Lemna sp.) obtained at Syngenta Crop Protection AG are

Table 1. Algal and macrophyte species compared in the sensitivity ranking

\begin{tabular}{|c|c|}
\hline Algae tested & Macrophytes tested \\
\hline $\begin{array}{l}\text { Anabaena flos-aquae } \\
\text { (Lyngb) Bréb. }\end{array}$ & Ceratophyllum demersum $\mathrm{L}$. \\
\hline $\begin{array}{l}\text { Chlamydomonas } \\
\text { reinhardii Dangeard }\end{array}$ & Elodea canadensis Michx. \\
\hline Chlorella pyrenoidosa Chick & Egeria densa Planch. \\
\hline Chlorella vulgaris Beijer. & $\begin{array}{l}\text { Glyceria maxima (Hartm.) } \\
\text { Holmb. }\end{array}$ \\
\hline Microcystis sp. Kütz. & $\begin{array}{l}\text { Hydrilla verticillata } \\
\text { (L.f.) Royle }\end{array}$ \\
\hline $\begin{array}{l}\text { Navicula pelliculosa } \\
\text { (Bréb.) Hilse }\end{array}$ & Lemna gibba $\mathrm{L}$. \\
\hline $\begin{array}{l}\text { Scenedesmus } \\
\text { quadricauda (Turp.) Bréb. }\end{array}$ & Lemna minor $\mathrm{L}$. \\
\hline $\begin{array}{l}\text { Scenedesmus } \\
\text { subspicatus } \text { Chodat }\end{array}$ & $\begin{array}{l}\text { Myriophyllum } \\
\text { heterophyllum Michx. }\end{array}$ \\
\hline $\begin{array}{l}\text { Selenastrum } \\
\text { capricornutum Printz }\end{array}$ & Myriophyllum spicatum $\mathrm{L}$. \\
\hline Skeletonema costatum Grev. & $\begin{array}{l}\text { Myriophyllum verticillatum } \mathrm{L} \text {. } \\
\text { Najas sp. L. } \\
\text { Potamogeton densus } \mathrm{L} .\end{array}$ \\
\hline
\end{tabular}

performed according to existing guidelines (ASTM, 1995; FIFRA, 1989a, b; OECD, 1984) or draft documents (OECD, 2001) already in discussion.

\section{Results}

Syngenta test results presented in Table 3 were performed in-house during the last months. A wider classification of the compounds than the one recommended by HRAC was performed based on their mode of action. The most sensitive species in the selected studies was identified and highlighted. The source of the data and the mode of action of specific compounds as well as a sensitivity ranking for the tested species based on median effect concentrations (EC50) were reported for each compound. The most sensitive species tested was identified, and taking this as a reference, the test species were grouped accordingly. Groupings were made according to EC50 values that differed by less than five times, less than 10 times and more than 10 times from the most sensitive species tested.

\section{Discussion}

Comparing plant species across the different experiments, the range of sensitivities identified show that there is no one plant species that is always the most sensitive, even for compounds with the same mode of action. It should also be noted that differences in the testing method (e.g., emerged, submersed or rooted form of the plant in the test, temperature, test medium, $\mathrm{pH}$, light intensity) or changes in the application method can lead to substantial differences in the values recorded as test endpoints. In the sensitivity ranking shown here, this problem is partly avoided because the comparison among species is made in the same study with the same testing method (see Fairchild et al., 1998 and Syngenta results). The difficulties related to data comparison between tests can be exemplified by the study of Fairchild et al. (1998), in which every alga was tested for $96 \mathrm{~h}$ with chlorophyll fluorescence as an endpoint while the duckweed Lemna sp. was tested over $96 \mathrm{~h}$ with the frond count as test endpoint. 


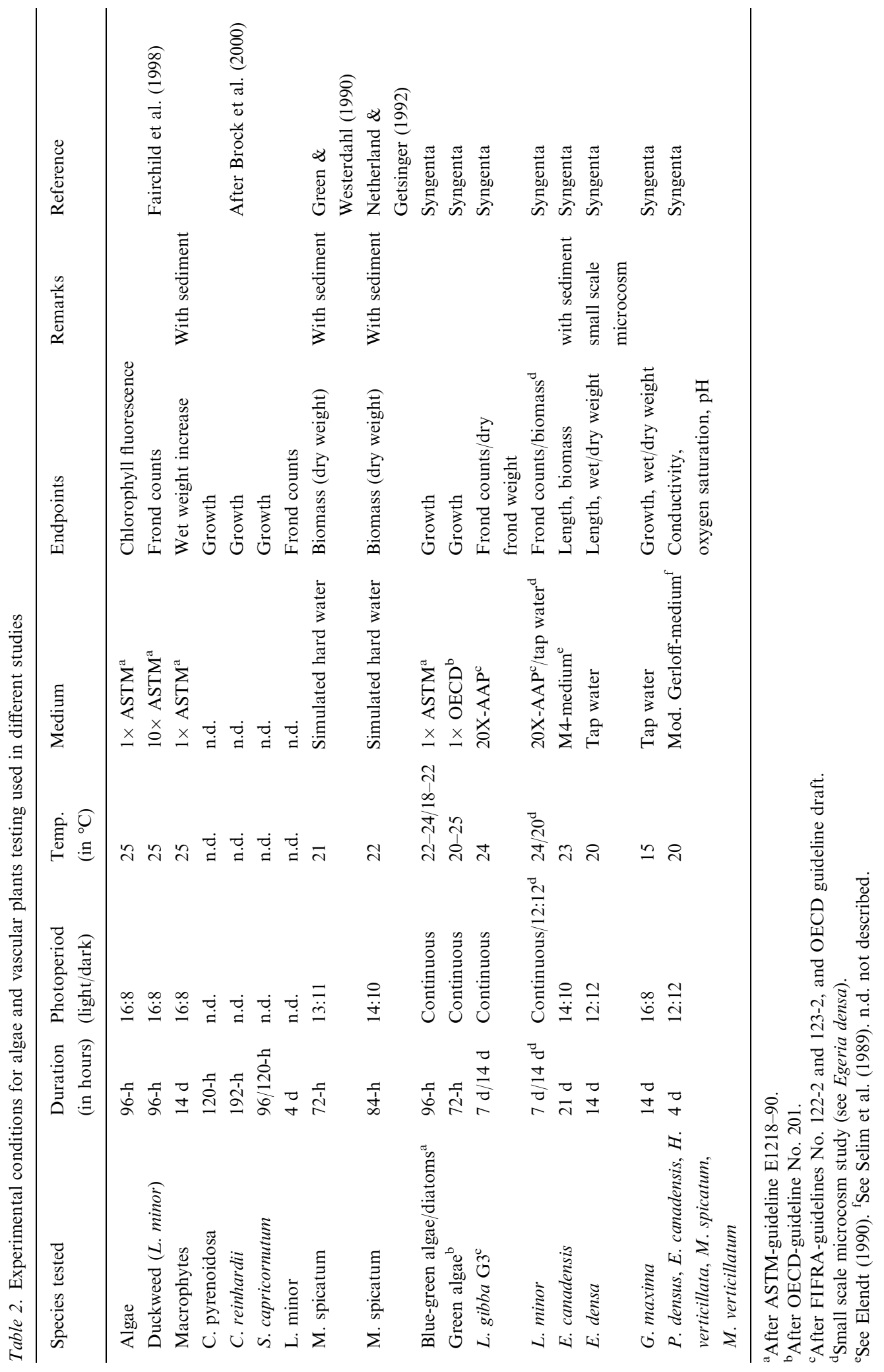


Table 3. Species sensitivity ranking for different herbicides with relation to their database and mode of action (vascular plants, algae); (required test species for preliminary risk assessment procedure (grey); most sensitive species (underlined))

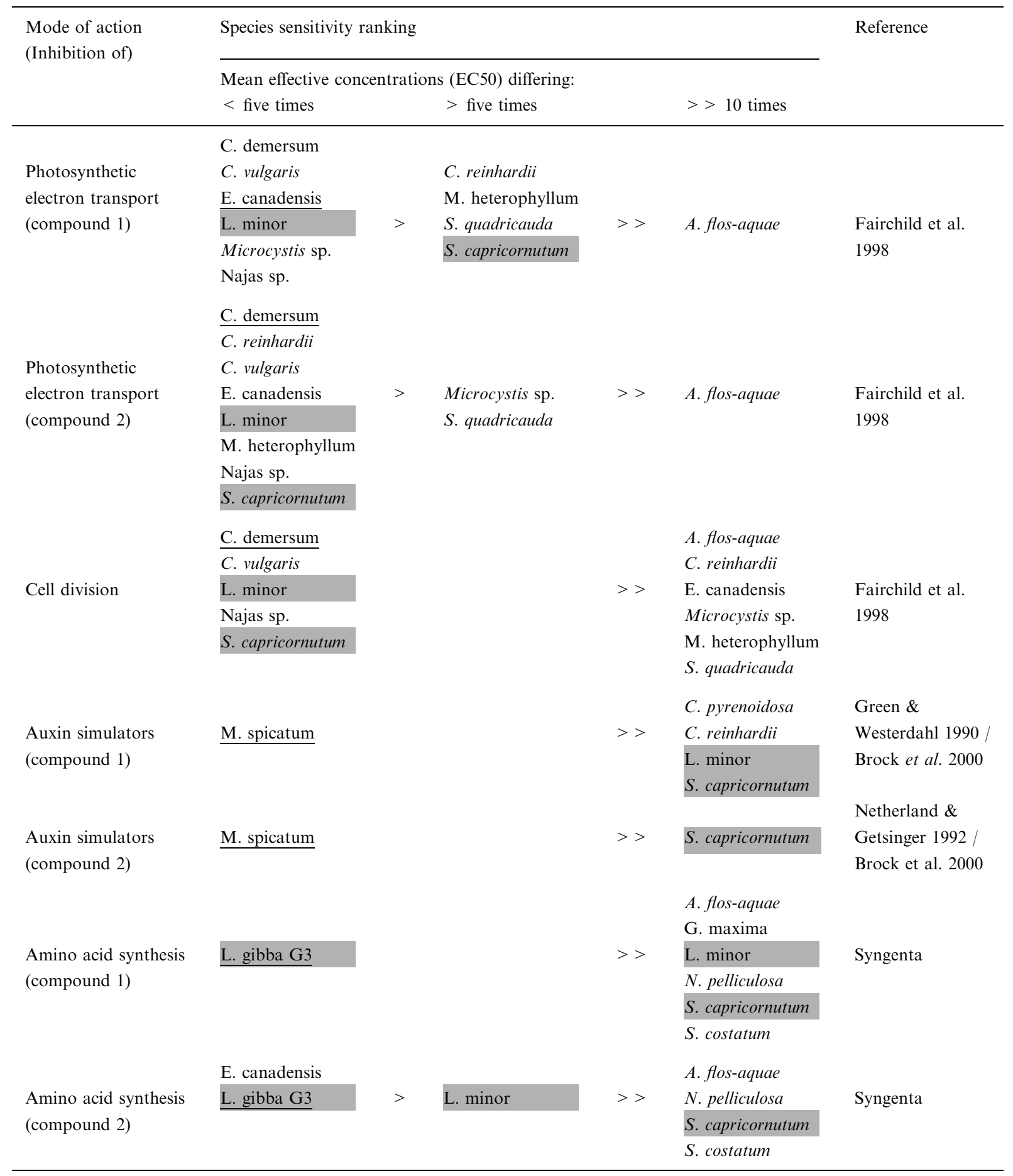


Table 3. (Continued)

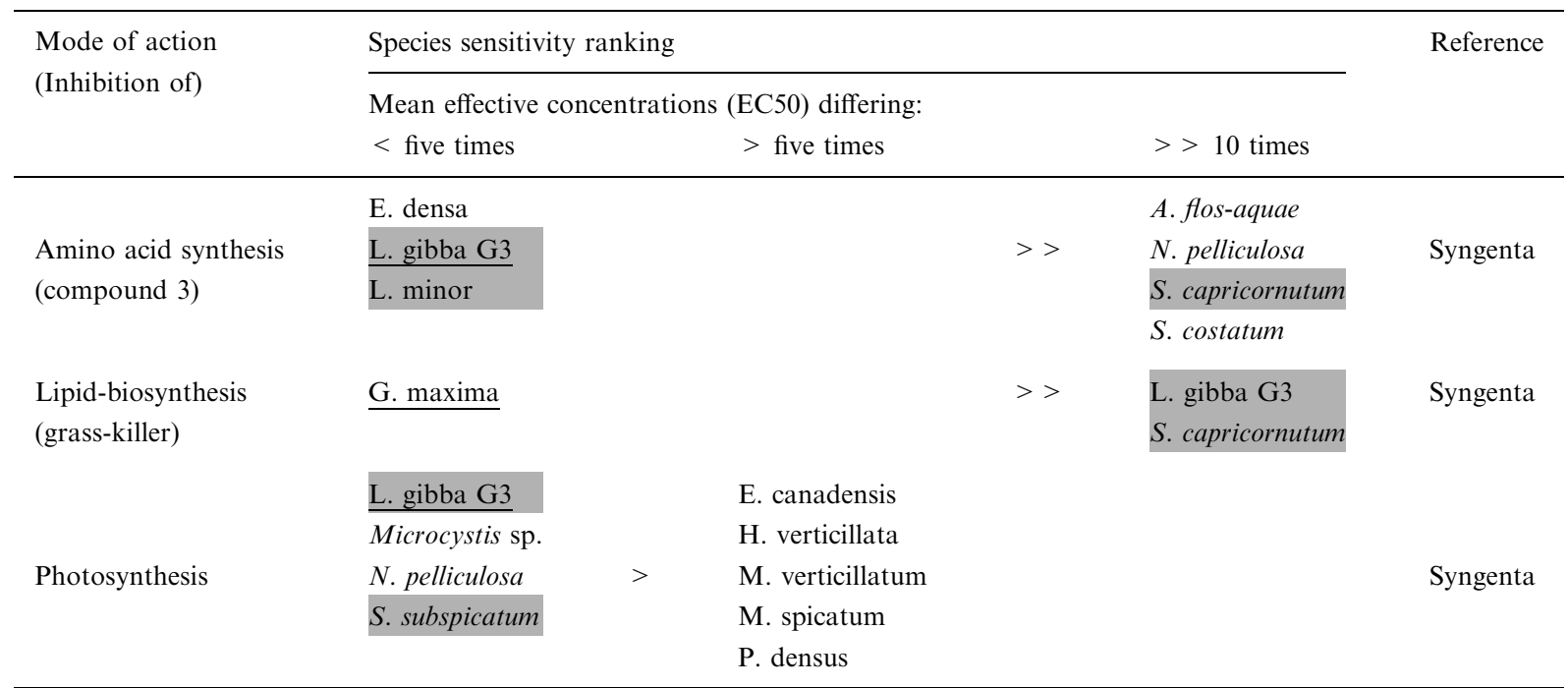

The other vascular plants were tested over 14 days with a measurement of wet weight increase. Consequently, robust comparisons of inherent sensitivity are difficult. For most of the existing studies, this particular issue is also a problem when comparing the results obtained in tests with the same plant species. For vascular plants in particular, this is an issue because there are no harmonised testing guidelines, test methods and endpoints are different. Even for species like the duckweed Lemna sp. where a guideline draft is available, there are still differences in methods. Only the testing of algal species is mostly performed according to the existing guidelines, which leads to comparable results due to standardisation of conditions and methodology. Brock et al. (2000) tried to solve the problem of comparing results from tests performed under different conditions by taking the geometric mean for the existing EC50 values of one species. In their report they compared the database available for algae, Lemna sp. and for a series of vascular plant species with respect to a list of nearly 20 compounds. They concluded that for over $80 \%$ of the compounds, the existing testing scheme with a green algae and Lemna sp. was sufficient to detect potential toxicity against non-target aquatic plants. What was apparent though, was that algae and Lemna sp. were inadequate for auxin simulating herbicides because in these tests the threshold value was underestimated up to a factor of 100. Auxin simulators generally appear to be more applicable to dicotyledonous macrophytes other than the monocot Lemna sp.

In our studies we always included Lemna sp. and green algae, which are test species required for the standard database in the preliminary risk assessment scheme. The results showed that the required test species produced EC50 values differing by less than five times from the most sensitive species. The only discernible exceptions were for two auxin simulating and a grass killing herbicide, where differences in the EC50 values between green algae and Lemna sp. were more than 10 times as compared to other vascular plants. For an acceptable risk assessment procedure, the existing testing scheme could be considered most protective under field conditions. However, this has to be proven by comparable results obtained under laboratory and field conditions using environmentally relevant concentrations.

At the moment, no international harmonised guidelines for aquatic plant testing exist and required testing protocols of different countries vary. Prior to the definition of further testing requirements by the US EPA we recommend to perform further investigations to define adequate test species and experimental designs (FIFRA SAP, 2001). 


\section{Conclusions}

The results presented by Brock et al. (2000) and our present comparison of literature and in-house data indicate that further test species need to be identified for testing the impact of auxin simulating herbicides or grass specific compounds to see if toxicity to non-target aquatic plants is underestimated.

Further studies will have to characterise adequate test species and to develop standardised experimental protocols before decisions on new regulations may be taken.

\section{Acknowledgements}

The research was mostly conducted at the facilities of Syngenta Crop Protection AG in Basel, Switzerland. We would like to thank Fabienne Walch, Delphine Alcantara-Gomez, Cecile Cazaubon and Hubert Dugon for their highly qualified technical assistance and support.

\section{References}

American Society for Testing and Materials (ASTM), 1995. Standard Guide for Conducting Static 96-h Toxicity Tests with Microalgae. E 1218-90, In Annual Book of ASTM Standards, Vol. 11.05, Philadelphia, PA, 573-584.

Brock, T. C. M., J. Lahr \& P. J. Van den Brink, 2000. Ecological Risks of Pesticides in Freshwater Ecosystems. Part 1:
Herbicides. Wageningen, Alterra, Green World Research. Alterra-Rapport 088.

Elendt, B. P., 1990. Selenium deficiency in crustacea; an ultrastructural approach to antennal damage in Daphnia magna Straus. Protoplasma 154: 25-33.

Fairchild, J. F., D. S. Ruessler \& A. R. Carlson, 1998. Comparative sensitivity of five species of macrophytes and six species of algae to atrazine, metribuzin, alachlor and metolachlor. Environmental Toxicology and Chemistry 17: 1830-1834.

FIFRA SAP (Federal Insecticide, Fungicide and Rodenticide Act Scientific Advisory Panel), 2001. Review of non-target plant toxicity tests under the North American Free Trade Agreement (NAFTA). SAP Report No. 2001-08, June 27-29, Arlington, VA.

FIFRA, 1989a. Guideline No. 122-2: Growth and Reproduction of Aquatic Plants - Tier I.

FIFRA, 1989b. Guideline No. 123-2: Growth and Reproduction of Aquatic Plants - Tier II.

Green, W. R. \& H. E. Westerdahl, 1990. Response of Eurasian watermilfoil to 2,4-D concentrations and exposure times. Journal of Aquatic Plant Management 28: 27-32.

Netherland, M. D. \& K. D. Getsinger, 1992. Efficacy of triclopyr on Eurasian watermilfoil: concentrations and exposure time effects. Journal of Aquatic Plant Management 30: $1-5$.

OECD (Organisation for Economic Cooperation and Development), 1984. Guideline-No. 201 for Testing of Chemicals: Alga, Growth Inhibition Test. Official Journal of the European Communities, 92/69/EEC: C.3.

OECD, 2001. Revised Draft Document: Lemna sp. Growth Inhibition Test.

Selim, S. A., S. W. O’Neal, M. A. Ross \& C. A. Lembi, 1989. Bioassay of photosynthetic inhibitors in water and aqueous soil extracts with Eurasian watermilfoil (Myriophyllum spicatum). Weed Science 37: 810-814.

US EPA, 2001. Proposal to Update Non-Target Plant Toxicity Testing under NAFTA. 